\title{
MedChemComm
}

Check for updates

Cite this: Med. Chem. Commun., 2017, 8, 2216

Received 7th April 2017,

Accepted 6th September 2017

DOI: $10.1039 / \mathrm{c} 7 \mathrm{md} 00170 \mathrm{c}$

rsc.li/medchemcomm

\section{Theoretical models of inhibitory activity for inhibitors of protein-protein interactions: targeting menin-mixed lineage leukemia with small molecules $\dagger$}

\author{
Wiktoria Jedwabny, ${ }^{a}$ Szymon Kłossowski, ${ }^{\text {b }}$ Trupta Purohit, ${ }^{\mathrm{b}}$ Tomasz Cierpicki, ${ }^{\mathrm{b}}$ \\ Jolanta Grembecka*b and Edyta Dyguda-Kazimierowicz (iD *a
}

\begin{abstract}
Development and binding affinity predictions of inhibitors targeting protein-protein interactions (PPI) still represent a major challenge in drug discovery efforts. This work reports application of a predictive nonempirical model of inhibitory activity for PPI inhibitors, exemplified here for small molecules targeting the menin-mixed lineage leukemia (MLL) interaction. Systematic ab initio analysis of menin-inhibitor complexes was performed, revealing the physical nature of these interactions. Notably, the non-empirical protein-ligand interaction energy comprising electrostatic multipole and approximate dispersion terms $\left(E_{\mathrm{El}, \mathrm{MTP}}^{(10)}+\right.$ $\left.E_{\text {Das }}\right)$ produced a remarkable correlation with experimentally measured inhibitory activities and enabled accurate activity prediction for new menin-MLL inhibitors. Importantly, this relatively simple and computationally affordable non-empirical interaction energy model outperformed binding affinity predictions derived from commonly used empirical scoring functions. This study demonstrates high relevance of the non-empirical model we developed for binding affinity prediction of inhibitors targeting protein-protein interactions that are difficult to predict using empirical scoring functions.
\end{abstract}

\section{Introduction}

Protein-protein interactions (PPI) between Mixed Lineage Leukemia (MLL) or MLL fusion proteins and menin are associated with a subtype of acute leukemia named MLL leukemia, ${ }^{1}$ which constitutes $5-10 \%$ of acute leukemia in adults ${ }^{2}$ and about $70 \%$ of acute leukemia in children. ${ }^{3}$ The treatment of MLL leukemia patients is currently ineffective, ${ }^{4}$ with a 5 year survival rate of about $35 \% .^{5}$ The menin-MLL proteinprotein interaction has been well validated as a therapeutic target in MLL leukemia using both genetic ${ }^{1}$ and pharmacologic approaches. ${ }^{6-11}$ Therefore, inhibition of the menin-MLL interaction appears to be of crucial importance for the development of novel targeted therapy against aggressive leukemia resulting from the leukemogenic activity of MLL fusion proteins.

\footnotetext{
${ }^{a}$ Department of Chemistry, Wroctaw University of Science and Technology, Wyb. Wyspiańskiego 27, 50-370 Wroctaw, Poland. E-mail: Edyta.Dyguda@pwr.edu.pl; Tel: +48713203200

${ }^{b}$ Department of Pathology, University of Michigan, $1150 \mathrm{~W}$. Medical Center Dr, MSRBI, Rm 4510D, Ann Arbor, MI, 48109, USA. E-mail: Jolantag@umich.edu; Fax: +734 763 8764; Tel: +734 6159319

$\dagger$ Electronic Supplementary Information (ESI) available: Detailed characterization of the compound collection; structural characteristics of the menin-MI-2-2 complex; and additional computational results. See DOI: 10.1039/c7md00170c
}

Our recently identified inhibitors disrupting the oncogenic activity of MLL fusion proteins by blocking the protein-protein interaction between menin and MLL belong to the thienopyrimidine class of compounds. ${ }^{6,7}$ These compounds directly bind to menin at MLL binding sites with low nanomolar binding affinities. We have also demonstrated that substantial improvement in inhibitory activity resulted from incorporation of fluorine atoms into an ethyl group on the thienopyrimidine scaffold. ${ }^{7}$ In particular, the MI-2-2 compound bearing a trifluoroethyl substituent exhibited an over 20-fold increase in the binding affinity compared to its nonfluorinated counterpart. ${ }^{6,7}$ Indeed, compounds with fluorine atoms are estimated to constitute about $20-25 \%$ of currently known drugs, as fluorine substituents affect both binding affinity and physicochemical properties of small molecules. ${ }^{12-14}$ While a rational approach for designing favorable fluorine interactions with proteins has recently been proposed, ${ }^{10}$ complementary methods providing insight into the physical nature of protein-ligand interactions would possibly augment these predictions. Furthermore, development of potent small molecule inhibitors targeting protein-protein interactions still constitutes a major challenge in the drug discovery field, ${ }^{15-17}$ and therefore identification of computational methods for effective prediction of binding affinities for PPI inhibitors represents a very important task and 
should attract a lot of attention in the drug discovery community.

Currently available methods used to estimate inhibitory activity are mostly based on empirical scoring functions or force fields, yielding widely different predictions. ${ }^{18,19}$ Due to significant computational cost, more accurate quantum chemical estimates are impractical in high throughput drug design and their applicability to protein-ligand interactions has been rather limited. ${ }^{20,21}$ However, alternative nonempirical models could be systematically derived by partitioning the $a b$ initio energy of the corresponding inhibitor-receptor interactions into well defined components using state-of-the-art perturbation or variational theories. ${ }^{22}$ Analytical formulas for separate binding energy terms can be the subject of subsequent approximations leading to affordable non-empirical scoring models of general applicability. We have previously shown that the electrostatic term alone was sufficient to rank polar or charged inhibitors of phenylalanine ammonia-lyase (PAL), ${ }^{22}$ leucine aminopeptidase (LAP) ${ }^{22}$ and urokinase (UPA). ${ }^{23}$ Further results characterizing essentially nonpolar inhibitors of fatty acid amide hydrolase $(\mathrm{FAAH})^{24}$ indicated that supplementing the electrostatic multipole term with the long-range dispersion contribution was required to afford reasonable correlation with experimental binding affinity data.

In contrast to empirical scoring functions derived from limited training sets, unified non-empirical models based on long-range multipole electrostatic and dispersion interactions might be more universal and less arbitrary. Assessment of the performance of non-empirical interaction energy analysis is especially important in the context of inhibitors targeting protein-protein interactions. Compared to widely studied enzyme-inhibitor interactions, experimental and computational characterization of small molecule PPI inhibition faces numerous challenges. ${ }^{25}$ Unlike buried binding cavities present in enzymes, binding sites at protein-protein interfaces are relatively flat and solvent exposed. ${ }^{26}$ Although several empirical and semi-empirical approaches have been successfully applied to rank selected PPI inhibitors, ${ }^{27-30}$ empirical scoring methods and knowledge-based potentials have not been trained to work with ligands that bind to PPI interfaces. ${ }^{31,32}$ Therefore, models of inhibitory activity derived from $a b$ initio quantum mechanical methods might then be particularly useful to describe the activity of PPI inhibitors more accurately as no parameterization is required here and the applicability of these methods might be more general.

This work aims to develop systematic non-empirical models of inhibitory activity for small molecule inhibitors blocking the protein-protein interaction between menin and MLL. The choice of the protein system and compounds was made to address the accuracy of description of PPI inhibition by small molecules using computational approaches. As already mentioned, we successfully applied this non-empirical model to FAAH inhibitors with the interaction energy being expressed by the long-range electrostatic and dispersion terms. ${ }^{24}$ To determine whether such a model can be applied to PPI inhibitors represents a major focus of this work.

The theoretical model of inhibitory activity reported here involves a representative model of the MLL binding site on menin (Fig. 1) and a set of menin-MLL inhibitors that we have already characterized experimentally ${ }^{6,7,10}$ (Table 1). To limit the computational cost required for reference $a b$ initio calculations of binding energy, only a subset of menin-MLL inhibitors reported in ref. 6, 7 and 10 was selected. Compound selection was made to accommodate the menin-MLL inhibitors with distinct substituents on the thienopyrimidine scaffold and a range of activity broad enough for the resulting model to be reliable. This system was used to develop a non-empirical model for activity prediction of menin-MLL inhibitors. Predictive capabilities of this approach were tested against new thienopyrimidine inhibitors of the menin-MLL interaction, developed independently to examine the influence of varying substituents to this scaffold on their inhibitory activity. Remarkably, nearly quantitative agreement was achieved between theoretically assessed and experimentally measured $\mathrm{IC}_{50}$ values. Competitive computational cost and more favorable performance of the nonempirical method applied here over those of commonly used empirical scoring functions indicate that this method can be successfully applied to rank newly designed inhibitors targeting protein-protein interactions.

\section{Methods}

\section{Physical nature of the menin-inhibitor interactions}

To analyze the physical nature of interactions between menin and small molecule inhibitors blocking the menin-MLL interaction, the binding energy was calculated according to the Hybrid Variational-Perturbation Theory (HVPT). ${ }^{33,34}$ This approach enables the study of relatively large systems (e.g.,

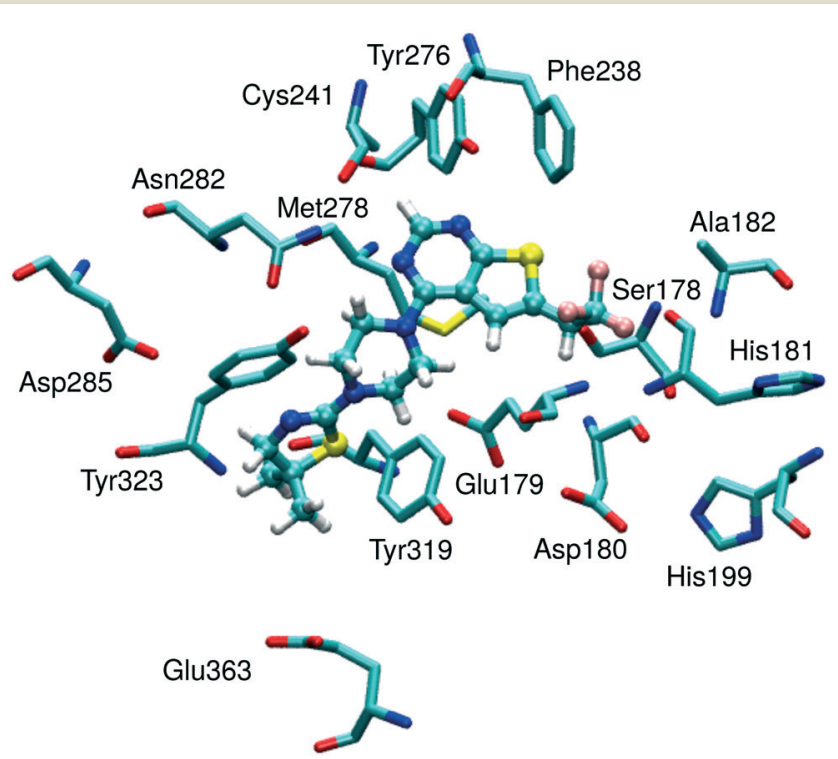

Fig. 1 Representative model of a menin binding site with an MI-2-2 inhibitor bound. The model was derived from the structure of the menin-MI-2-2 complex (4GQ4 in PDB). 
Table 1 Structures and experimental activity ${ }^{6,7,10}$ of inhibitors targeting menin-MLL interaction

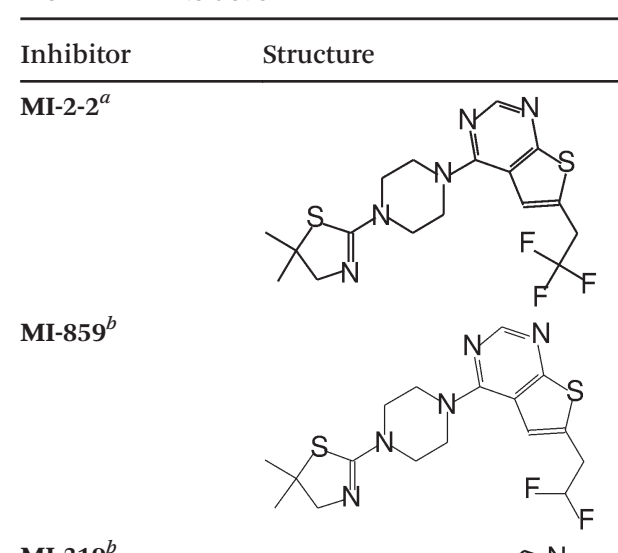

MI-319 $^{b}$<smiles>FC(F)c1nnc(N2CCN(c3ncnc4sc(CC(F)(F)F)cc34)CC2)s1</smiles>

MI-2-3 ${ }^{b}$<smiles>FC(F)(F)Cc1cc2c(N3CCN(c4nnc(C(F)(F)F)s4)CC3)ncnc2s1</smiles>

MI-836 $^{b}$<smiles>CC1(C)CN=C(N2CCN(c3ncnc4sc(CCF)cc34)CC2)S1</smiles>

${\mathrm{MI}-2^{c}}^{c}$<smiles>CCCc1cc2c(N3CCN(C4=NCC(C)(C)S4)CC3)ncnc2s1</smiles>

$\mathrm{MI}-273^{b}$<smiles>CC1(C)CN=C(N2CCN(C3=NCNC4SC(C(F)(F)F)=CC34)CC2)S1</smiles>

MI-20 ${ }^{c}$<smiles>CCCCc1cc2c(N3CCN(C4=NCC(C)(C)S4)CC3)ncnc2s1</smiles>

MI-2-4 ${ }^{a}$<smiles>CC(c1cc2c(N3CCN(C4=NCC(C)(C)S4)CC3)ncnc2s1)C(F)(F)F</smiles>

MI-326 ${ }^{b}$<smiles>Cc1nnc(N2CCN(c3ncnc4sc(CC(F)(F)F)cc34)CC2)s1</smiles>

0.065

0.092

0.260

0.45

0.674
Table 1 (continued)

\begin{tabular}{lll}
\hline Inhibitor & Structure \\
\hline $\mathbf{M I}-19^{b}$ & $\mathrm{IC}_{50}[\mu \mathrm{M}]$ \\
\hline
\end{tabular}

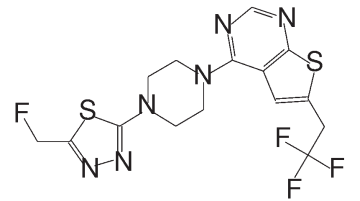

MI-12 ${ }^{c}$

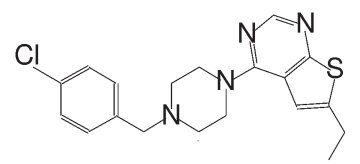

MI-16 ${ }^{c}$<smiles>CCc1cc2c(N3CCN(c4nccs4)CC3)ncnc2s1</smiles>

46<smiles>CCc1cc2c(N3CCN(Cc4ccccc4)CC3)ncnc2s1</smiles>

MI-10 ${ }^{c}$<smiles>CCc1cc2c(N3CCN(c4ccccn4)CC3)ncnc2s1</smiles>

MI-11 $^{c}$<smiles>CCc1cc2c(N3CCN(c4ncccn4)CC3)ncnc2s1</smiles>

1.653

4

MI-4 ${ }^{c}$

MI-6<smiles>CCc1cc2c(N3CCN(c4nncs4)CC3)ncnc2s1</smiles>

193

${ }^{a}$ Inhibitory activity values are taken from ref. $7 .{ }^{b}$ Inhibitory activity values are taken from ref. $10{ }^{c}$ Inhibitory activity values are taken from ref. 6.

enzyme-inhibitor complexes modelled as representative sets of protein amino acid residues and the corresponding ligands) at a reasonable computational cost. ${ }^{22,35-39}$ The HVPT decomposition scheme of the interaction energy defines partitioning of the second-order Møller-Plesset (MP2) binding energy $\left(E_{\mathrm{MP} 2}\right)$ into the electrostatic multipole $\left(E_{\mathrm{EL}, \mathrm{MTP}}^{(10)}\right)$, penetration $\left(E_{\mathrm{EL}, \mathrm{PEN}}^{(10)}\right)$, exchange $\left(E_{\mathrm{EX}}^{(10)}\right)$, delocalization $\left(E_{\mathrm{DEL}}^{(\mathrm{RO})}\right)$ and correlation $\left(E_{\mathrm{CORR}}^{(2)}\right)$ terms, as shown in eqn (1): 


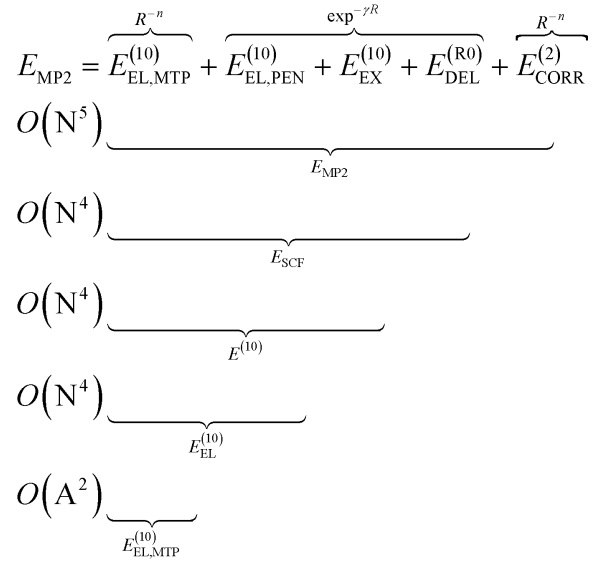

The subsequent contributions to the MP2 binding energy are characterized by increasing computational cost, as indicated by $O(X)$ scaling, where $\mathrm{N}$ and A stand for the number of atomic orbitals and atoms, respectively (see eqn (1)).

The $E_{\mathrm{EL}, \mathrm{MTP}}^{(10)}$ term in eqn (1) represents the long-range electrostatic multipole binding energy calculated from atomic multipole expansion. ${ }^{40}$ This term includes interactions of Mulliken atomic charges in addition to interactions of atomic dipoles, quadrupoles, hexadecapoles, etc., representing the anisotropy of atomic charge distribution.

The short-range penetration term $E_{\mathrm{EL}, \mathrm{PEN}}^{(10)}$ is defined as $E_{\mathrm{EL}, \mathrm{PEN}}^{(10)}$ $=E_{\mathrm{EL}}^{(10)}-E_{\mathrm{EL}, \mathrm{MTP}}^{(10)}$, where $E_{\mathrm{EL}}^{(10)}$ represents the first-order electrostatic energy. The first-order repulsive exchange term $E_{\mathrm{EX}}^{(10)}$ is calculated from the first-order Heitler-London energy, $E^{(10)}: E_{\mathrm{EX}}^{(10)}=$ $E^{(10)}-E_{\mathrm{EL}}^{(10)}$. The classical induction and charge transfer terms are represented by the higher order delocalization energy, $E_{\mathrm{DEL}}^{(\mathrm{RO})}$, which is obtained as the difference between the counterpoisecorrected self-consistent field (SCF) variational energy, $E_{\mathrm{SCF}}$, and the first-order Heitler-London energy, $E^{(10)}$. The correlation term, accounting for dispersion and exchange-dispersion interactions as well as the intramolecular correlation contribution, is defined as: $E_{\mathrm{CORR}}^{(2)}=E_{\mathrm{MP} 2}-E_{\mathrm{SCF}}$. As emphasized in eqn (1), all the subsequent corrections to $E_{\mathrm{MP} 2}$ interaction energy could be categorized into long- and short-range interactions varying with the intermolecular distance, $R$, as $R^{-n}(n \in \mathbb{N})$ and $\exp ^{-\gamma R}(\gamma>0)$, respectively. In the above equations, the zero value of the second superscript represents uncorrelated interaction energy contributions, and the $E_{\mathrm{CORR}}^{(2)}$ term denotes the inter- and intra-molecular correlation contributions.

Within the HVPT decomposition scheme, the dispersion component is included in the computationally demanding $E_{\text {CORR }}^{(2)}$ correlation energy term, scaling with the fifth power of molecular size expressed by the number of atomic orbitals, $\mathrm{N}$. The recently derived atom-atom potential function, $E_{\text {Das }}$, fitted to benchmark values of dispersion interactions ${ }^{41,42}$ has been a far more affordable alternative to these costly calculations, as they scale with the square number of atoms $O\left(\mathrm{~A}^{2}\right)$, in contrast to $a b$ initio calculations scaling at least with the fifth power of the number of orbitals, $O\left(\mathrm{~N}^{5}\right)$. Following the remarkable performance of the $E_{\text {Das }}$ function to describe noncovalent interactions in hydrogen-bonded complexes, ${ }^{43}$ we applied this approximate dispersion term in our model for inhibitory activity prediction.

\section{Calculation of binding energy}

The structures of menin-MLL inhibitors considered herein are given in Table 1. The crystal structure of menin in complex with the MI-2-2 inhibitor (PDB accession code $4 \mathrm{GQ4} ;^{7}$ $1.27 \AA$ resolution) was used for modeling of the geometry of the remaining complexes. The structures of inhibitors were built using Schrödinger Suite 2012 (ref. 44) and further refined by all-atom optimization in the Maestro ${ }^{44}$ implemented OPLS 2005 force field, ${ }^{45}$ with the convergence set to a nonhydrogen atom RMSD of $0.1 \AA$. The preparation of the protein structure involved: (i) determination of the optimal hydrogen bonding network for the protein crystal structure with Protein Preparation Wizard ${ }^{46}$ PROPKA implementation, ${ }^{47-50}$ at $\mathrm{pH} 7.5$, i.e. the $\mathrm{pH}$ matching the experimental conditions for the measurements of inhibitory activities, (ii) building of missing hydrogen atoms with Maestro, and (iii) optimization of the hydrogen atoms in Maestro and the OPLS 2005 force field, following the optimization protocol provided by Protein Preparation Wizard (heavy atoms were kept frozen and the convergence criterion was defined as the hydrogen atom RMSD of $0.3 \AA$ A). The same protein receptor structure was used for the following binding energy calculations and scoring of all the menin-MLL inhibitors considered herein. Nonempirical interaction energy evaluation involved a limitedsize model of the receptor, composed of selected amino acid residues, as described in the following discussion.

Binding energy calculations involved a menin binding site represented by 15 amino acid residues (Fig. 1) selected by their closest proximity to the ligand, namely within approximately 4 A from MI-2-2 (see Table S1, ESI, $\dagger$ for the distances between MI-2-2 and menin residues). Negatively charged Asp180 and positively charged His199 residues constituted an ionic pair, and such a pair was included in further calculations (i.e., as a neutral Asp180-His199 dimer formed by counter-charged residues). There were no counter-charged amino acid residues in close proximity to the remaining negatively charged residues (Glu179, Asp285 and Glu363). The dangling bonds arising from cutting the residues out of the protein structure were saturated by hydrogen atoms.

The menin-inhibitor binding energy was calculated as the sum of the above defined intermolecular energy components obtained for each inhibitor-amino acid residue pair with a modified $^{34}$ version of GAMESS $^{51}$ program using the 6-31G(d $)^{52-54}$ basis set. Counterpoise correction was applied to eliminate the basis set superposition error. ${ }^{55}$

\section{Binding affinity predictions using empirical scoring functions}

To perform empirical scoring with the menin-inhibitor complexes obtained in a way described in the previous section, the following functions implemented in Discovery Studio 3.5 
Suite $^{56}$ were used: LigScore, ${ }^{57}$ Piecewise Linear Potential (PLP) ${ }^{58,59}$ Jain, $^{60}$ Potential of Mean Force (PMF), ${ }^{61,62}$ Ludi, ${ }^{63,64}$ and Discovery Studio binding free energy. ${ }^{65}$ Two commonly used docking programs, AutoDock Vina ${ }^{66}$ and GOLD 4.0, ${ }^{67}$ were also employed in the scoring of the enzyme-inhibitor interactions. Calculations performed with AutoDock Vina and GOLD involved no docking to avoid the influence of the docking procedure on the scoring results. All available Gold scoring functions were tested, i.e., GoldScore, ChemScore, and Astex Statistical Potential (ASP). PyMOL ${ }^{68}$ and the PyMOL AutoDock/Vina plugin ${ }^{69}$ were used for the preparation of the receptor and inhibitors for scoring in AutoDock Vina. The latter was carried out with a 22.5 A cubic grid centered on the MI-2-2 inhibitor. A GOLD scoring run was performed with a spherical grid centered in the same way and encompassing the amino acid residues within $15.0 \AA$ radius from the point of origin.

The performance of particular scoring methods was evaluated by means of the Pearson's correlation coefficient calculated with respect to experimentally determined inhibitory activity $^{6,7,10}$ (see Table 1). Scoring functions, for which the higher score indicates the greater binding potency, were assigned to the opposite of the calculated value of the correlation coefficient to enable direct comparison with the results of non-empirical binding energy calculations, assigning lower interaction energy values to more potent inhibitors. The results were additionally compared in terms of the success rate of prediction, $N_{\text {pred }}$ (the predictivity). Following nonparametric statistics, the predictivity refers to the percentage of concordant pairs among all possible pairs of complexes within a given set. A concordant pair denotes two complexes with computationally evaluated relative stability being of the same sign as the relative experimental binding affinity (see ref. 70 for details).

\section{Development of novel menin-MLL inhibitors}

The development and synthesis of compounds: MI-2-2, MI859, MI-319, MI-2-3, MI-836, MI-2, MI-273, MI-20, MI-2-4, MI326, MI-19, MI-333, MI-12, MI-16, MI-4, MI-10, MI-11, and MI-6 which were used to develop the theoretical model of inhibitory activity, was described before. ${ }^{6,7,10}$ Novel menin-MLL inhibitors (compounds 1, 2, 3, 4, 5, 6, and 7) were designed using the menin-MI-2-2 complex ${ }^{7}$ to test the effect of varying substituents on the MI-2-2 scaffold. These new compounds were obtained from synthesis on demand from the contract research organization. All compounds were provided with the certificate of analysis and in addition were fully characterized in-house by MS and NMR (see the ESI $\dagger$ for characterization of compounds $1,2,3,4,5,6$, and 7 ).

\section{Biological activity of menin-MLL inhibitors}

The inhibitory activity of compounds: MI-2-2, MI-859, MI-319, MI-2-3, MI-836, MI-2, MI-273, MI-20, MI-2-4, MI-326, MI-19, MI-333, MI-12, MI-16, MI-4, MI-10, MI-11, and MI-6, was reported before. ${ }^{6,7,10}$ Inhibition of the menin-MLL interac- tion by compounds 1-7 was assessed by the fluorescence polarization (FP) assay using the protocol described previously. $^{6,71}$ Briefly, the fluorescein-labeled MLL (MBM1) peptide at $10 \mathrm{nM}$, menin at $100 \mathrm{nM}$ and compounds at varying concentrations were used for $\mathrm{IC}_{50}$ determination in $\mathrm{FP}$ buffer (50 mM Tris, pH 7.5, $50 \mathrm{mM} \mathrm{NaCl,} 1 \mathrm{mM}$ DTT). The compounds (5\% final DMSO concentration) were added to the menin-MLL peptide complex and incubated for $1 \mathrm{~h}$ before changes in fluorescence polarization were measured using a PHERAstar microplate reader (BMG).

\section{Results and discussion}

\section{Interaction energy calculations for menin-inhibitor complexes}

We performed the $a b$ initio quantum mechanics calculations of the non-empirical interaction energy between menin and the thienopyrimidine class of our recently developed meninMLL inhibitors with inhibitory activity spanning over the five orders of magnitude (Table 1). Eighteen compounds were initially selected to calculate their interaction energy with the representative model of the menin binding site comprising 15 amino acid residues positioned in the closest proximity to the ligand molecules (Fig. 1). The total binding energy values at the subsequent levels of theory, obtained as a sum of the interaction energy values calculated in a pairwise manner for each amino acid residue on menin and the corresponding ligand molecule, for all menin-inhibitor complexes are listed in Table 2. The total interaction energy calculated at the highest level of theory, $E_{\mathrm{MP} 2}$ (eqn (1)), has negative values for all inhibitors included in this study, ranging from -37.8 to $-26.8 \mathrm{kcal} \mathrm{mol}^{-1}$ (Table 2), which supports their favorable interactions with menin. The dominant component of the total interaction energy is the electrostatic term $E_{\mathrm{EL}}^{(10)}$, which changes from -49.6 to $-41.0 \mathrm{kcal} \mathrm{mol}^{-1}$, supporting the importance of the electrostatic contribution to the ligand binding affinity for this class of menin-MLL inhibitors. The positive values of the $E^{(10)}$ energy, originating from the repulsive $E_{\mathrm{EX}}^{(10)}$ term, suggest the presence of short contacts between inhibitors and binding site residues. ${ }^{23} E_{\mathrm{SCF}}$ energy values are also mostly positive, but substantially smaller than $E^{(10)}$ values due to stabilizing delocalization interactions. Finally, the correlation term $\left(E_{\mathrm{CORR}}^{(10)}\right)$ restores the stabilizing characteristics reflected by the negative values of the total interaction energy calculated at the reference MP2 level of theory. The interaction energy estimate composed of the multipole component of the electrostatic energy and the approximate dispersion term $\left(E_{\mathrm{EL}, \mathrm{MTP}}^{(10)}+E_{\mathrm{Das}}\right)$, has resulted in the lowest (the most negative) values (Table 2) due to the lack of repulsive contributions in this particular energy expression.

For all menin-inhibitor complexes included in this study, Tyr276 serves as a hydrogen bond donor interacting with the nitrogen atom of the thienopyrimidine ring in the meninMLL inhibitors. Compared to the remaining residues, Tyr276 significantly contributes to the total interaction energy of all inhibitors (Table S2, ESI $\dagger$ ). The interaction energy values 
Table 2 Total menin-inhibitor interaction energy ${ }^{a}$ at the consecutive levels of theory

\begin{tabular}{|c|c|c|c|c|c|c|}
\hline Inhibitor & $E_{\mathrm{EL}, \mathrm{MTP}}^{(10)}$ & $E_{\mathrm{EL}}^{(10)}$ & $E^{(10)}$ & $E_{\mathrm{SCF}}$ & $E_{\mathrm{MP} 2}$ & $E_{\mathrm{EL}, \mathrm{MTP}}^{(10)}+E_{\mathrm{Das}}$ \\
\hline MI-859 & -25.0 & -48.8 & 22.9 & 5.4 & -35.3 & -98.0 \\
\hline MI-836 & -25.9 & -49.6 & 20.4 & 2.8 & -37.8 & -96.8 \\
\hline MI-2 & -23.3 & -45.9 & 21.9 & 3.4 & -36.8 & -94.7 \\
\hline MI-273 & -22.1 & -49.3 & 31.9 & 14.5 & -28.0 & -101.8 \\
\hline MI-326 & -26.2 & -47.2 & 17.9 & 1.1 & -36.7 & -97.4 \\
\hline MI-19 & -22.1 & -44.1 & 22.8 & 5.0 & -34.8 & -92.3 \\
\hline MI-333 & -26.8 & -48.2 & 17.9 & 1.0 & -36.9 & -97.4 \\
\hline MI-12 & -25.7 & -43.9 & 11.7 & -3.5 & -34.6 & -80.9 \\
\hline MI-16 & -19.3 & -41.7 & 22.4 & 6.4 & -30.3 & -80.9 \\
\hline MI-4 & -23.4 & -41.6 & 14.0 & -1.0 & -32.4 & -78.5 \\
\hline$S E^{d}$ & 2.1 & 1.6 & 5.2 & 5.1 & 2.9 & 3.9 \\
\hline
\end{tabular}

${ }^{a}$ In units of kcal mol ${ }^{-1} \cdot{ }^{b}$ Percentage of successful predictions [\%]. ${ }^{c}$ Correlation coefficient for the correlation of the energy obtained at a given level of theory and the experimental inhibitory activity expressed as $\mathrm{FC}_{50} \cdot{ }^{d}$ Standard error of estimate, in units of kcal mol ${ }^{-1}$.

associated with essentially all Tyr276-inhibitor pairs fluctuate around -15 and $-6 \mathrm{kcal} \mathrm{mol}^{-1}$ for $E_{\mathrm{EL}}^{(10)}$ and $E_{\mathrm{MP} 2}$ energy, respectively, indicating the presence of strong hydrogen bonding in all menin-inhibitor complexes studied here. In addition, Asn282, Met278, and Glu179 also appear to have a pronounced impact on inhibitor binding affinities due to their substantial contribution to the total binding energy (Table S2, ESI $\dagger$ ). The involvement of the remaining menin residues in inhibitor binding seems to be less significant.

\section{Correlation of interaction energy with inhibitory activity of menin-MLL inhibitors}

The highest absolute values of $E_{\mathrm{MP} 2}$ and $E_{\mathrm{EL}}^{(10)}$ were obtained for the most potent inhibitors described here (Table 2). Likewise, the weakest MI-6 inhibitor is characterized by the lowest absolute values of $E_{\mathrm{MP} 2}$ and $E_{\mathrm{EL}}^{(10)}$ energies, suggesting a possible correlation with the ligand binding affinity. To quantify the relationship between the interaction energy and inhibitory activity, the interaction energy values calculated at different levels of theory were correlated with the $\mathbf{I C}_{50}$ values of menin-MLL inhibitors measured using the fluorescence polarization assay (Table 1). The relationship between the total interaction energies calculated at selected levels of theory and the inhibitory activity of respective inhibitors is plotted in Fig. 2.

Very strong correlation was obtained for $E_{\mathrm{EL}}^{(10)}$ energy, resulting in a remarkably high correlation coefficient $R$, equal to -0.87 (Fig. 2). Furthermore, the electrostatic multipole term $E_{\mathrm{EL}, \mathrm{MTP}}^{(10)}$ also demonstrates correlation with the inhibitory activity of menin-MLL inhibitors $(R=-0.63$; Fig. 2). These results indicate that the electrostatic interaction energy appears to be sufficient to represent the differences in the menin-inhibitor binding affinities within this class of compounds. This is likely related to the nature of the protein binding site, as the inhibitor binding pocket on menin features a number of polar amino acid residues. Indeed, only four out of 15 residues included in these calculations possess non-polar side chains, while the remaining 11 amino acid residues bear polar or charged side chains (e.g., Glu179, Asp285, Glu363, Asp180, His199, Ser178, and Asn282; see Fig. 1). Accordingly, the dominant contribution of the electrostatic effects in the interaction energy is reflected by its good correlation with the binding affinity characterizing these compounds.

In contrast, the remaining components of the interaction energy, i.e., $E^{(10)}$ and $E_{\mathrm{SCF}}$ energy terms, do not exhibit

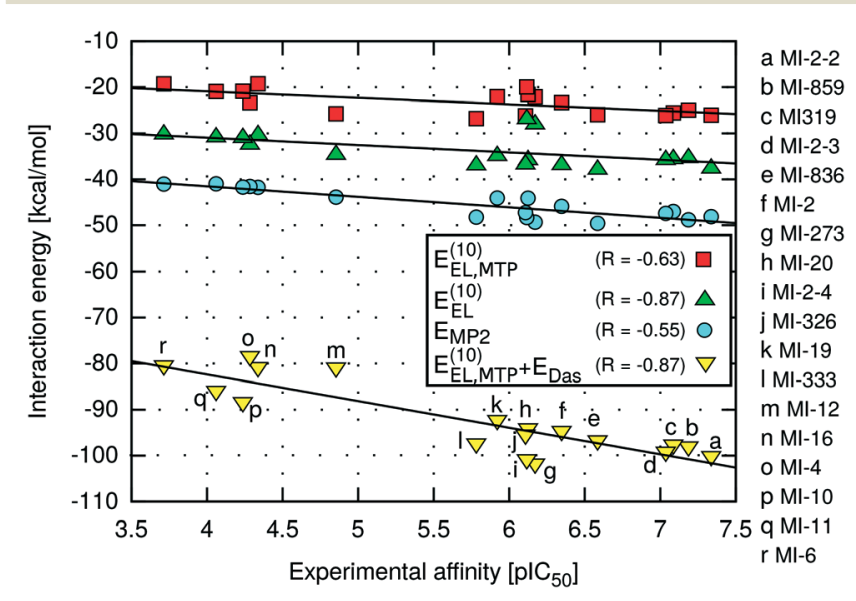

Fig. 2 Menin-inhibitor binding energies at different levels of theory as a function of inhibitory activity. 
satisfactory correlation with the inhibitory activity of the thienopyrimidine class of menin-MLL inhibitors (Table 2). The lack of correlation between the inhibitory activity and $E^{(10)}$ interaction energy presumably arises from including the short-range interaction energy component, which is very sensitive to any inaccuracies that might occur in the modelled protein-ligand complexes. The repulsive exchange term depends exponentially on the intermolecular distance and shortening of the intermonomer separation has a large impact on the corresponding binding energy. ${ }^{23,72}$ Recent systematic analysis of 66 biomolecular complexes ${ }^{72}$ indicated that considering only the long-range electrostatic term (without taking into account the repulsive exchange term) leads to much better estimates of relative ligand binding affinities. More importantly, this phenomenon is valid even for shortened intermolecular distances resulting from force field inadequacy $^{73}$ or the basis set superposition error. ${ }^{74}$ Interestingly, it has been shown that augmenting the multipole electrostatic term with $E_{\text {Das }}$ dispersion approximation can further improve predictions of relative stabilities within a particular set of protein-ligand complexes. ${ }^{24}$

Indeed, despite the prevalence of electrostatic interactions in menin-inhibitor complexes, the $E_{\mathrm{EL}, \mathrm{MTP}}^{(10)}+E_{\text {Das }}$ model, in which both electrostatic and dispersion interactions are incorporated, is characterised by the strong correlation with the inhibitory activity, reflected by the high value of the correlation coefficient ( $R=-0.87$; Fig. 2 ). Therefore, it seems that the interactions of predominantly electrostatic nature could be accurately and inexpensively described if the dispersion effects are incorporated in addition to the electrostatic term. Moreover, the significantly improved predictivity of $E_{\mathrm{EL}, \mathrm{MTP}}^{(10)}+E_{\mathrm{Das}}$ compared to that of $E_{\mathrm{MP} 2}$ energy is probably due to the absence of the repulsive exchange term, $E_{\mathrm{EX}}^{(10)}$, which is included at the MP2 level of theory. As mentioned above, this particular term is very sensitive to the presence of intermolecular short contacts commonly occurring in modelled structures of protein-ligand complexes. ${ }^{23,72}$ In fact, the preparation of the menin-inhibitor complexes applied herein involved a simple optimization procedure employing rigid receptor representation. More sophisticated refinement protocols were avoided to verify the applicability of the proposed approach to quick assessment of the structural modifications in, e.g., the lead optimization stage. Possible unresolved steric clashes have probably resulted in the overly magnified repulsive exchange term, $E_{\mathrm{EX}}^{(10)}$, and the loss of correlation at the higher levels of theory (up to the $E_{\mathrm{MP} 2}$ energy), which include this particular interaction energy contribution.

In general, dispersion interactions are not absolutely required to properly describe the inhibitor binding forces within systems with dominant electrostatic interactions, such as menin-inhibitor complexes. However, a simple electrostatic model of interaction energy is clearly insufficient in the case of nonpolar hydrophobic systems. ${ }^{24}$ The performance of the $E_{\mathrm{EL}, \mathrm{MTP}}^{(10)}+E_{\text {Das }}$ model proposed herein appears to be satisfactory regardless of the physical nature of the inhibitory activity, and thus such a model could be used universally in the inhibitor design process without an unnecessary increase in the computational cost.

Differences in ligand binding affinities might arise from the interactions of inhibitors with a subset of amino acid residues in the binding site. The significant contribution to the total binding energy does not necessarily indicate that a given amino acid residue is responsible for the observed inhibitory activity differences, as such interaction could be similar for all tested inhibitors. Residues that contribute the most to the relative inhibitory activity might be identified by monitoring the changes in correlation coefficients upon removal of particular residues from the binding site model (Table S3, ESI $\dagger$ ). Accordingly, the interaction of inhibitors with Glu179, Asp285, Tyr276, and Tyr323 residues seems to be the most important in terms of the differences in the binding potencies. Upon removal of these residues from the model of the menin binding site, the values of the corresponding correlation coefficients become substantially worse (Table S3, ESI $\dagger$ ). Therefore, the interaction with Glu179, Asp285, Tyr276, and Tyr323 residues appears to influence the relative binding affinities and should be carefully studied while designing novel menin-MLL inhibitors.

\section{Scoring with empirical functions}

We have also applied a number of empirical scoring approaches available in the Discovery Studio, ${ }^{56}$ GOLD $4.0,{ }^{67}$ and AutoDock Vina ${ }^{66}$ programs to compare their predictive capability with the quantum mechanics-based models presented in Table 2. The performances of these empirical scoring methods, reflected by the correlation of the calculated binding affinity estimate with the experimentally measured inhibitory activity, are compared in Table 3. Overall, the empirical scoring functions tested herein yielded varying predictions with respect to the experimentally established ranking of the menin-MLL inhibitors. Only four out of 15 scoring functions exhibited favorable predictive capabilities, as demonstrated by the $R$ correlation coefficient and $N_{\text {pred }}$ (defined as the percentage of successful predictions) values exceeding or close to 0.8 and $75 \%$, respectively. The best performance was obtained for the LigScore1 scoring function implemented in Discovery Studio $\left(R=-0.81, N_{\text {pred }}=75.2 \%\right.$; Table 3$)$. However, the performance of the majority of the remaining empirical scoring approaches evaluated here is unsatisfactory, with poor correlation reflected by unsatisfactory $\mathrm{R}$ values $(R<0.7)$ and insufficient predictivity (Table 3 ). Utterly different predictive abilities of empirical scoring functions might originate from the inherent parameterization carried out with training sets, which are rarely applicable to all protein-ligand complexes. Besides, selection of the best performing scoring function for new designed ligands represents a challenge due to high variability of the results obtained from various scoring functions applied. An additional challenge posed here is the fact that we are dealing with inhibitors of protein-protein interactions. Distinct features of binding pockets existing in such complexes may not be accounted for by the empirical 
Table 3 Performance of various empirical scoring methods for ranking the menin-MLL inhibitors (results for the non-empirical $E_{\mathrm{EL}, \mathrm{MTP}}^{(10)}+E_{\mathrm{Das}}$ model are provided for comparison)

\begin{tabular}{llc}
\hline Scoring function & $R^{a}$ & $N_{\text {pred }}{ }^{a}$ \\
\hline$E_{\mathrm{EL}, \mathrm{MTP}}^{(10)}+E_{\text {Das }}$ & -0.87 & 81.1 \\
LigScore1 & -0.81 & 75.2 \\
Jain & -0.80 & 77.8 \\
$E_{\text {binding }}$ (Discovery Studio 3.5) & -0.79 & 74.5 \\
PLP2 & -0.79 & 80.4 \\
PLP1 & -0.74 & 77.8 \\
PMF04 & -0.65 & 73.2 \\
Ludi2 & -0.62 & 72.6 \\
LigScore2 & -0.43 & 69.9 \\
Ludi1 & -0.40 & 58.8 \\
Ludi3 & -0.23 & 54.3 \\
PMF & +0.24 & 41.2 \\
& & \\
Goldscore & -0.64 & 69.9 \\
ASP & -0.62 & 70.6 \\
Chemscore & -0.28 & 60.1 \\
Binding affinity (AutoDock Vina) & -0.67 & 73.2 \\
${ }^{a}$ Correlation coefficient between the calculated binding & affinity \\
estimate and the experimental inhibitory activity expressed as $p \mathrm{IC}_{50}$. \\
${ }^{b}$ Percentage of successful predictions [\%]. &
\end{tabular}

scoring methods calibrated with classical protein-ligand interactions, such as enzyme-inhibitor complexes. ${ }^{31,32}$

While plausible predictions were obtained with selected empirical scoring approaches, the $E_{\mathrm{EL}, \mathrm{MTP}}^{(10)}+E_{\mathrm{Das}}$ model of inhibitory activity appears to outperform the classical scoring functions presented herein, resulting in remarkable agreement with the experimental data characterized by $R=-0.87$ and $N_{\text {pred }}$ $=81.1 \%$. The computational cost of the $E_{\mathrm{EL}, \mathrm{MTP}}^{(10)}+E_{\text {Das }}$ function is as attainable as that of empirical scoring models. ${ }^{24}$ Unlike the latter, no parameterization employing empirical data was involved to develop the $E_{\mathrm{EL}, \mathrm{MTP}}^{(10)}+E_{\text {Das }}$ model. Successful prediction of the relative binding affinities of menin-MLL inhibitors suggests that small molecule inhibitors that block protein-protein interactions are within the range of applicability of this particular non-empirical scoring method.

\section{Binding affinity predictions of novel menin-MLL inhibitors}

We then aimed to use the non-empirical interaction energy calculations to predict the binding affinity of new meninMLL inhibitors. Rapid prediction of the inhibitory activity of novel menin-MLL inhibitors can be made with the following relationship derived from the linear least-squares regression analysis of experimental $p \mathrm{IC}_{50}$ data and calculated $E_{\mathrm{EL}, \mathrm{MTP}}^{(10)}+$ $E_{\text {Das }}$ values (the latter are given in $\mathrm{kcal} \mathrm{mol}^{-1}$ ), resulting in the following equation:

$$
p \mathrm{IC}_{50}=\frac{E_{\mathrm{EL}, \mathrm{MTP}}^{(10)}+E_{\mathrm{Das}}+59.28}{-5.78}
$$

To examine the applicability of this method to predict the activity of new menin-MLL inhibitors, seven novel com- pounds with varying substituents on the thienopyrimidine scaffold of MI-2-2 were selected. The development, synthesis and characterization of these compounds were performed as a part of the structure-activity studies directed at optimization of the lead compound. The structures and inhibitory activities of seven novel menin-MLL inhibitors are presented in Table 4. With the exception of the least potent compound 7 , the range of inhibitory activity characterizing these novel menin-MLL inhibitors is similar to the reference set of compounds listed in Table 1 . The highest potency was obtained for compounds 1 and $2\left(\mathrm{IC}_{50}=193 \mathrm{nM}\right.$ and $650 \mathrm{nM}$, respectively), while other compounds showed inhibitory activity in the micromolar range (Table 4).

Table 4 Structures and experimental activity of novel inhibitors targeting menin-MLL interaction

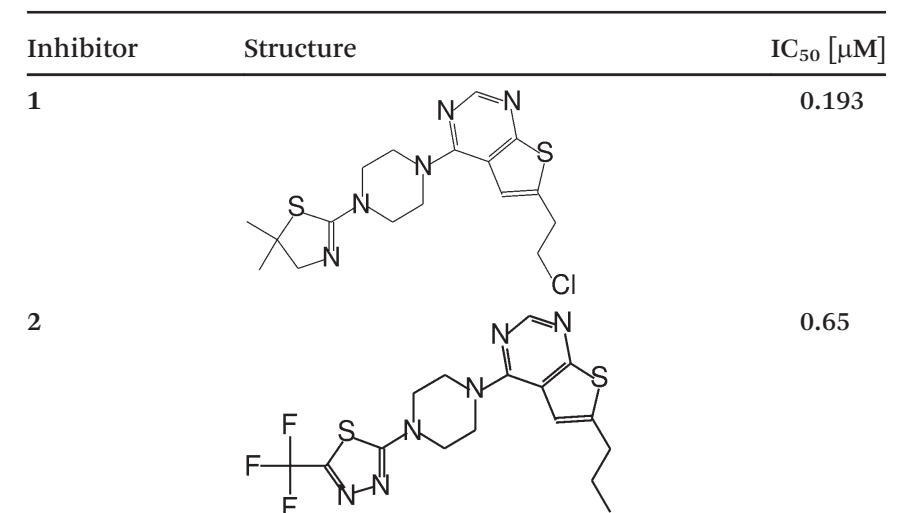

3<smiles>CCc1cc2c(N3CCN(c4nnc(C(F)(F)F)s4)CC3)ncnc2s1</smiles><smiles>FC(F)(F)Cc1cc2c(N3CCN(c4nnc(C(F)(F)F)o4)CC3)ncnc2s1</smiles>

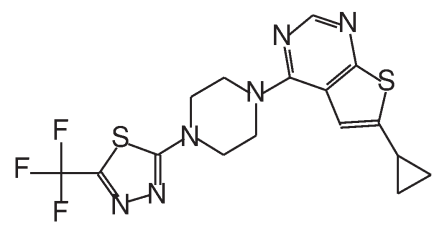<smiles>CSc1cc2c(N3CCN(c4nnc(C(F)(F)F)s4)CC3)ncnc2s1</smiles>

20.00<smiles>CCCc1cc2c(N3CCN(C4=NNC(C(F)(F)F)N4)CC3)ncnc2s1</smiles> 
The predictive capabilities of eqn (2) were then tested against the set of novel menin-MLL inhibitors (Table 4), and the calculated interaction energy for the new compounds was used to predict their theoretical $\mathrm{IC}_{50}$ values. The systematic analysis of the interaction energy values calculated at the subsequent levels of theory for these seven menin-MLL complexes is provided in Table S4, ESI.† As observed in the case of the initial set of 18 menin-MLL inhibitors (Table 2), the $E_{\text {MP2 }}$ binding energy is characterized by negative values, ranging from -34.5 to $-30.5 \mathrm{kcal} \mathrm{mol}^{-1}$ (Table S4, ESI $\dagger$ ). The interactions formed by these new menin-MLL inhibitors are essentially electrostatic in nature, which is reflected by $E_{\mathrm{EL}}^{(10)}$ binding energy values exceeding $-35 \mathrm{kcal} \mathrm{mol}^{-1}$. Accounting for the repulsive $E_{\mathrm{EX}}^{(10)}$ term, possibly overestimated due to shortened intermonomer distances, ${ }^{23}$ results in positive values of both $E^{(10)}$ and the subsequent $E_{\mathrm{SCF}}$ binding energy terms. The delocalization and correlation contributions are both required to restore the stabilizing nature of the interaction energy obtained at the reference MP2 level of theory. The correlation between the calculated $E_{\mathrm{MP} 2}$ binding energy and the experimental binding potency is reflected by the correlation coefficient $R=-0.65$ (Table S4, ESI $\dagger$ ). Noticeably, the $R$ values of -0.83 and -0.84 characterize the electrostatic term, $E_{\mathrm{EL}}^{(10)}$, and the $E_{\mathrm{EL}, \mathrm{MTP}}^{(10)}+E_{\mathrm{Das}}$ energy values, respectively, confirming the prevalence of electrostatic interactions at the inhibitor-protein interface and the adequacy of the $E_{\mathrm{EL}, \mathrm{MTP}}^{(10)}+E_{\text {Das }}$ function for the description of menin-MLL inhibition.

The performance of empirical scoring functions already considered for the training set of menin-MLL inhibitors (Table 3) was also tested on the set of seven inhibitors analyzed herein. The predictive capabilities of these empirical models are presented in Table S5, ESI. $\dagger$ Similarly to the results obtained in the training set of 18 inhibitors (Table 3), only two of the empirical scoring approaches considered here yielded predictions characterized with $R$ correlation coefficients exceeding or close to 0.8 (Table S5, ESI $\dagger$ ). The best estimates of the experimental inhibitory activity of seven meninMLL inhibitors were obtained with the LigScore2 scoring function implemented in Discovery Studio $(R=-0.84$ and $N_{\text {pred }}=66.7 \%$; Table S5, ESI $\dagger$ ). Most of the empirical scoring models tested herein exhibited either weak or no agreement with the experimental data. The fact that all but one of the inhibitors within the test set are fluorine-rich compounds might contribute to the poor performance of the empirical functions when applied to this particular set. Unlike most of the empirical approaches, the non-empirical $E_{\mathrm{EL}, \mathrm{MTP}}^{(10)}+E_{\text {Das }}$ model also appears to exhibit satisfactory predictive capabilities over the test set $\left(R=-0.84\right.$ and $N_{\text {pred }}=66.7 \%$; Table S4, $\mathrm{ESI} \dagger$ ).

As already noted, the $E_{\mathrm{EL}, \mathrm{MTP}}^{(10)}+E_{\mathrm{Das}}$ energy was employed for the prediction of binding potency of 7 new menin-MLL inhibitors according to eqn (2). The comparison of the calculated and experimental $p \mathrm{IC}_{50}$ values for new menin-MLL inhibitors is provided in Fig. 3, resulting in a very good correlation described by $R=-0.84$. As shown in Fig. 3, the inhibitory activity of all these compounds reported here is

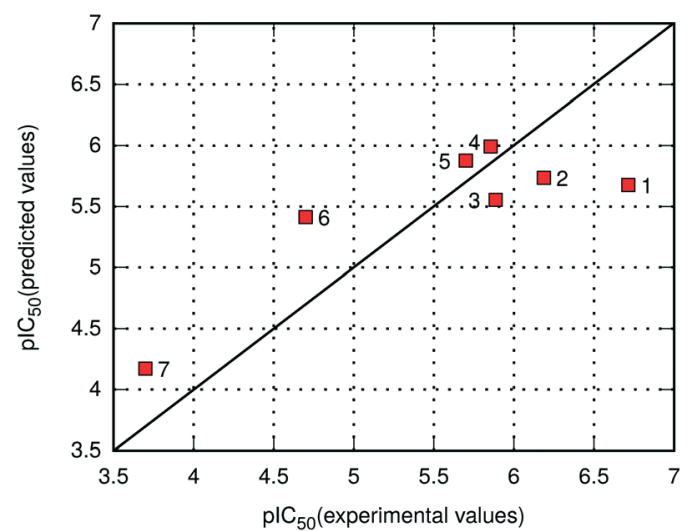

Fig. 3 Predicted values of $\mathrm{pIC}_{50}$ for novel menin-MLL inhibitors.

predicted with nearly quantitative accuracy. Compounds 1-5 are relatively similar in terms of their binding potency (Table 4). When applied to the inhibition constant, an order of magnitude change is associated with approximately 1.3 kcal mol $^{-1}$ increment in the free energy of binding. ${ }^{75}$ Therefore, it would not be reasonable to expect that such small differences in the $p \mathrm{IC}_{50}$ values might be very accurately captured by any computational method, as they exceed the accuracy of most quantum chemical calculations. Accordingly, the performance of the $E_{\mathrm{EL}, \mathrm{MTP}}^{(10)}+E_{\text {Das }}$ scoring function in predicting the inhibitory activity of novel menin-MLL inhibitors is adequate, with the binding potency of these compounds reproduced with nearly quantitative agreement. Considering that six out of seven inhibitors discussed here are fluorinerich compounds, these results also suggest that the $E_{\mathrm{EL}, \mathrm{MTP}}^{(10)}+$ $E_{\text {Das }}$ approach is capable of capturing the changes in the binding properties resulting from fluorine substitutions. Overall, very good agreement between the experimental and theoretical data presented herein demonstrates the usefulness and applicability of the non-empirical interaction energy calculations for successful predictions of inhibitory activity of menin-MLL inhibitors and possibly other inhibitors targeting protein-protein interactions.

\section{Conclusions}

Binding affinity prediction for newly designed protein inhibitors still represents a challenge, in particular for the more complex systems, such as inhibitors of protein-protein interactions. Here, we applied quantum chemical calculations of the interaction energy for the complexes of menin-MLL inhibitors to formulate and test the non-empirical model of inhibitory activity. Systematic analysis of the binding energy calculated at the consecutive levels of theory and comparison of the interaction energy with inhibitory activity of the menin-MLL inhibitors resulted in a strong correlation obtained for the $E_{\mathrm{EL}}^{(10)}$ interaction energy component, indicating that electrostatic interactions are very important in the 
system. Furthermore, our studies resulted in the validation of a simplified model, the $E_{\mathrm{EL}, \mathrm{MTP}}^{(10)}+E_{\mathrm{Das}}$ non-empirical function comprising long-range interaction energy components, namely the electrostatic multipole and dispersion contributions. This model showed satisfactory correlation between the calculated interaction energy and experimentally measured inhibitory activity for the menin-MLL inhibitors. Importantly, the non-empirical $E_{\mathrm{EL}, \mathrm{MTP}}^{(10)}+E_{\text {Das }}$ model outperformed numerous empirical scoring functions tested here for comparison. As computationally inexpensive electrostatic multipole and approximate dispersion terms are employed herein, such a non-empirical function could become a computational approach as affordable as widely used empirical scoring methods for activity prediction of protein inhibitors, including inhibitors of protein-protein interactions.

As revealed by the analysis of the interaction energy, binding of menin-MLL inhibitors is essentially electrostatic in nature. The non-empirical $E_{\mathrm{EL}, \mathrm{MTP}}^{(10)}+E_{\text {Das }}$ model has already been proven to be applicable to mostly nonpolar enzyme-inhibitor complexes. ${ }^{24}$ This contribution indicates the relevance of the $E_{\mathrm{EL}, \mathrm{MTP}}^{(10)}+E_{\text {Das }}$ model to polar or charged complexes. The range of applicability of this particular model for binding affinity prediction seems to encompass inhibitors of proteinprotein interactions and ligands with multiple fluorine substitutions. Finally, the successful prediction of inhibitory activity of new menin-MLL inhibitors validates the usefulness of the $E_{\mathrm{EL}, \mathrm{MTP}}^{(10)}+E_{\text {Das }}$ model in the structure-based development of novel inhibitors, including inhibitors of protein-protein interactions, for which majority of empirical scoring functions provide rather poor predictions of ligand binding affinity. As demonstrated by nearly quantitative agreement between the predicted and experimental binding potency within the validation set of inhibitors, the $E_{\mathrm{EL}, \mathrm{MTP}}^{(10)}+E_{\text {Das }}$ model allows for the inexpensive and reliable assessment of the influence of structural changes made to the parent scaffold on the inhibitory activity of the resulting compounds. Further computational efforts will be directed at determination of the applicability of the $E_{\mathrm{EL}, \mathrm{MTP}}^{(10)}+E_{\text {Das }}$ model for testing of prospective PPI inhibitors characterized with more extensive structural differences.

\section{Conflicts of interest}

Drs. Grembecka and Cierpicki receive research support from Kura Oncology Inc. They also receive compensation as members of the Scientific Advisory Board of Kura Oncology, and they have equity ownership in the company. Other co-authors declare no potential conflict of interest.

\section{Acknowledgements}

This project was supported by the National Institute of Health (NIH) grants R01 CA160467 to J. G. and R01 CA181185 to T. C., LLS Scholar (1215-14) to J. G. and American Cancer Society
Research Scholar grant (RSG-13-130-01-CDD) to J. G. This project was financed in part by a statutory activity subsidy from the Polish Ministry of Science and Higher Education for the Faculty of Chemistry of Wrocław University of Science and Technology and in part by the Wrocław Research Centre EIT+ within the project "Biotechnologies and advanced medical technologies" - BioMed (POIG.01.01.02-02-003/08) co-financed by the European Regional Development Fund (Operational Programme Innovative Economy, 1.1.2). We also thank Wrocław University of Science and Technology and the University of Michigan for support. Calculations were performed at the Wrocław Supercomputer and Networking Center (WCSS). Discovery Studio calculations were carried out with Bioviva Polish Academic Country Wide licence.

\section{References}

1 A. Yokoyama, T. C. P. Somervaille, K. S. Smith, O. Rozenblatt-Rosen, M. Meyerson and M. L. Cleary, Cell, 2005, 123, 207-218.

2 R. Marschalek, Br. J. Haematol., 2011, 152, 141-154.

3 D. Tomizawa, K. Koh, T. Sato, N. Kinukawa, A. Morimoto, K. Isoyama, Y. Kosaka, T. Oda, M. Oda, Y. Hayashi, M. Eguchi, K. Horibe, T. Nakahata, S. Mizutani and E. Ishii, Leukemia, 2007, 21, 2258-2263.

4 R. K. Slany, Hematol. Oncol., 2005, 23, 1-9.

5 J. F. DiMartino and M. L. Cleary, Br. J. Haematol., 1999, 106, 614-626.

6 J. Grembecka, S. He, A. Shi, T. Purohit, A. G. Muntean, R. J. Sorenson, H. D. Showalter, M. J. Murai, A. M. Belcher, T. Hartley, J. L. Hess and T. Cierpicki, Nat. Chem. Biol., 2012, 8, 277-284.

7 A. Shi, M. J. Murai, S. He, G. Lund, T. Hartley, T. Purhoit, G. Reddy, M. Chruszcz, J. Grembecka and T. Cierpicki, Blood, 2012, 120, 4461-4469.

8 S. He, T. J. Senter, J. Pollock, C. Han, S. K. Upadhyay, T. Purohit, R. D. Gogliotti, C. W. Lindsley, T. Cierpicki, S. R. Stauffer and J. Grembecka, J. Med. Chem., 2014, 57, 1543-1556.

9 D. Borkin, S. He, H. Miao, K. Kempinska, J. Pollock, J. Chase, T. Purohit, B. Malik, T. Zhao, J. Wang, B. Wen, H. Zong, M. Jones, G. Danet-Desnoyers, M. L. Guzman, M. Talpaz, D. L. Bixby, D. Sun, J. L. Hess, A. G. Muntean, I. Maillard, T. Cierpicki and J. Grembecka, Cancer Cell, 2015, 27, 589-602.

10 J. Pollock, D. Borkin, G. Lund, T. Purohit, E. DygudaKazimierowicz, J. Grembecka and T. Cierpicki, J. Med. Chem., 2015, 58, 7465-7474.

11 D. Borkin, J. Pollock, K. Kempinska, T. Purohit, X. Li, B. Wen, T. Zhao, H. Miao, S. Shukla, M. He, D. Sun, T. Cierpicki and J. Grembecka, J. Med. Chem., 2016, 59, 892-913.

12 H. J. Böhm, D. Banner, S. Bendels, M. Kansy, B. Kuhn, K. Muller, U. Obst-Sander and M. Stahl, ChemBioChem, 2004, 5, 637-643. 
13 K. Mueller, C. Faeh and F. Diederich, Science, 2007, 317, 1881-1886.

14 S. Purser, P. R. Moore, S. Swallow and V. Gouverneur, Chem. Soc. Rev., 2008, 37, 320-330.

15 D. P. Ryan and J. M. Matthews, Curr. Opin. Struct. Biol., 2005, 15, 441-446.

16 T. Cierpicki and J. Grembecka, Immunol. Rev., 2015, 263, 279-301.

17 G. Zinzalla and D. E. Thurston, Future Med. Chem., 2009, 1, 65-93.

18 A. R. Leach, B. K. Shoichet and C. E. Peishoff, J. Med. Chem., 2006, 49, 5851-5855.

19 D. Plewczyński, M. Łaźniewski, R. Augustyniak and K. Ginalski, J. Comput. Chem., 2011, 32, 742-755.

20 N. D. Yilmazer and M. Korth, J. Phys. Chem. B, 2013, 117, 8075-8084.

21 U. Ryde and P. Söderhjelm, Chem. Rev., 2016, 116, 5520-5566.

22 E. Dyguda, J. Grembecka, W. A. Sokalski and J. Leszczyński, J. Am. Chem. Soc., 2005, 127, 1658-1659.

23 R. Grzywa, E. Dyguda-Kazimierowicz, M. Sieńczyk, M. Feliks, W. A. Sokalski and J. Oleksyszyn, J. Mol. Model., 2007, 13, 677-683.

24 W. Giedroyć-Piasecka, E. Dyguda-Kazimierowicz, W. Beker, M. Mor, A. Lodola and W. A. Sokalski, J. Phys. Chem. B, 2014, 118, 14727-14736.

25 M. R. Arkin, Y. Tang and J. A. Wells, Chem. Biol., 2014, 21, 1102-1114.

26 R. J. Bienstock, Curr. Pharm. Des., 2012, 18, 1240-1254.

27 Z.-Y. Jiang, M.-C. Lu, L.-L. Xu, T.-T. Yang, M.-Y. Xi, X.-L. Xu, X.-K. Guo, X.-J. Zhang, Q.-D. You and H.-P. Sun, J. Med. Chem., 2014, 57, 2736-2745.

28 H. Li, H. Xiao, L. Lin, D. Jou, V. Kumari, J. Lin and C. Li, J. Med. Chem., 2014, 57, 632-641.

29 J. Chen, J. Wang, Q. Zhang, K. Chen and W. Zhu, Sci. Rep., 2015, 5, 17421.

30 W. Huang, L. Cai, C. Chen, X. Xie, Q. Zhao, X. Zhao, H.-Y. Zhou, B. Han and C. Peng, J. Biomol. Struct. Dyn., 2016, 34, 341-351.

31 L. Laraia, G. McKenzie, D. R. Spring, A. R. Venkitaraman and D. J. Huggins, Chem. Biol., 2015, 22, 689-703.

32 M. A. Kuenemann, O. Sperandio, C. M. Labbé, D. Lagorce, M. A. Miteva and B. O. Villoutreix, Prog. Biophys. Mol. Biol., 2015, 119, 20-32.

33 W. A. Sokalski, S. Roszak and K. Pecul, Chem. Phys. Lett., 1988, 153, 153-159.

34 R. W. Góra, W. A. Sokalski, J. Leszczyński and V. Pett, J. Phys. Chem. B, 2005, 109, 2027-2033.

35 J. Grembecka, P. Kedzierski and W. A. Sokalski, Chem. Phys. Lett., 1999, 313, 385-392.

36 P. Kedzierski, W. A. Sokalski and M. Krauss, J. Comput. Chem., 2000, 21, 432-445.

37 W. A. Sokalski, P. Kedzierski and J. Grembecka, Phys. Chem. Chem. Phys., 2001, 3, 657-663.

38 B. Szefczyk, A. J. Mulholland, K. E. Ranaghan and W. A. Sokalski, J. Am. Chem. Soc., 2004, 126, 16148-16159.
39 K. M. Langner, P. Kedzierski, W. A. Sokalski and J. Leszczyński, J. Phys. Chem. B, 2006, 110, 9720-9727.

40 W. A. Sokalski and A. Sawaryn, J. Chem. Phys., 1987, 87, 526-534.

41 K. Pernal, R. Podeszwa, K. Patkowski and K. Szalewicz, Phys. Rev. Lett., 2009, 103, 263201-263204.

42 R. Podeszwa, K. Pernal, K. Patkowski and K. Szalewicz, J. Phys. Chem. Lett., 2010, 1, 550-555.

43 J. Hoja, A. Sax and K. Szalewicz, Chem. - Eur. J., 2014, 20, 2292-2300.

44 Schrödinger Suite 2012, Maestro version 9.3, Schrödinger, LLC, New York, NY, 2012.

45 J. L. Banks, H. S. Beard, Y. Cao, A. E. Cho, W. Damm, R. Farid, A. K. Felts, T. A. Halgren, D. T. Mainz, J. R. Maple, R. Murphy, D. M. Philipp, M. P. Repasky, L. Y. Zhang, B. J. Berne, R. A. Friesner, E. Gallicchio and R. M. Levy, J. Comput. Chem., 2005, 26, 1752-1780.

46 Schrödinger Suite 2012, Protein Preparation Wizard: Epik 2.3, Impact 5.8, Prime 3.1, Schrödinger, LLC, New York, NY, 2012.

47 H. Li, A. D. Robertson and J. H. Jensen, Proteins, 2005, 61, 704-721.

48 D. C. Bas, D. M. Rogers and J. H. Jensen, Proteins, 2008, 73, 765-783.

49 M. H. M. Olsson, C. R. Søndergard, M. Rostkowski and J. H. Jensen, J. Chem. Theory Comput., 2011, 7, 525-537.

50 C. R. Søndergard, M. H. M. Olsson, M. Rostkowski and J. H. Jensen, J. Chem. Theory Comput., 2011, 7, 2284-2295.

51 M. W. Schmidt, K. K. Baldridge, J. A. Boatz, S. T. Elbert, M. S. Gordon, J. H. Jensen, S. Koseki, N. Matsunaga, K. A. Nguyen, S. J. Su, T. L. Windus, M. Dupuis and J. A. Montgomery, J. Comput. Chem., 1993, 14, 1347-1363.

52 P. C. Hariharan and J. A. Pople, Theor. Chim. Acta, 1973, 28, 213-222.

53 M. M. Francl, W. J. Pietro, W. J. Hehre, J. S. Binkley, D. J. DeFrees, J. A. Pople and M. S. Gordon, J. Chem. Phys., 1982, 77, 3654-3665.

54 V. A. Rassolov, J. A. Pople, M. A. Ratner and T. L. Windus, J. Chem. Phys., 1998, 109, 1223-1229.

55 S. Boys and F. Bernardi, Mol. Phys., 1970, 19, 553-566.

56 Accelrys Software Inc., Discovery Studio Modeling Environment, Release 3.5, 2012.

57 A. Krammer, P. D. Kirchhoff, X. Jiang, C. M. Venkatachalam and M. Waldman, J. Mol. Graphics Modell., 2005, 23, 395-407.

58 D. K. Gehlhaar, G. M. Verkhivker, P. A. Rejto, C. J. Sherman, D. B. Fogel, L. J. Fogel and S. T. Freer, Chem. Biol., 1995, 2, 317-324.

59 D. K. Gehlhaar, D. Bouzida and P. A. Rejto, in Reduced Dimensionality in Ligand-Protein Structure Prediction: Covalent Inhibitors of Serine Proteases and Design of Site-Directed Combinatorial Libraries, American Chemical Society, 1999, ch. 19, vol. 719, pp. 292-311.

60 A. N. Jain, J. Comput.-Aided Mol. Des., 1996, 10, 427-440.

61 I. Muegge and Y. C. Martin, J. Med. Chem., 1999, 42, 791-804.

62 I. Muegge, J. Med. Chem., 2006, 49, 5895-5902. 
63 H. J. Böhm, J. Comput.-Aided Mol. Des., 1994, 8, 243-256.

64 H. J. Böhm, J. Comput.-Aided Mol. Des., 1998, 12, 309-323.

65 J. Tirado-Rives and W. L. Jorgensen, J. Med. Chem., 2006, 49, 5880-5884.

66 O. Trott and A. J. Olson, J. Comput. Chem., 2010, 31, 455-461.

67 G. Jones, P. Willett, R. C. Glen, A. R. Leach and R. Taylor, J. Mol. Biol., 1997, 267, 727-748.

68 PyMOL(TM) Molecular Graphics System, Version 1.5.0.1., Schrödinger, LLC.

69 D. Seeliger and B. L. de Groot, J. Comput.-Aided Mol. Des., 2010, 24, 417-422.
70 K. M. Langner, W. Beker and W. A. Sokalski, J. Phys. Chem. Lett., 2012, 3, 2785-2789.

71 J. Grembecka, A. M. Belcher, T. Hartley and T. Cierpicki, J. Biol. Chem., 2010, 285, 40690-40698.

72 W. Beker, K. M. Langner, E. Dyguda-Kazimierowicz, M. Feliks and W. A. Sokalski, J. Comput. Chem., 2013, 34, 1797-1799.

73 R. S. Paton and J. M. Goodman, J. Chem. Inf. Model., 2009, 49, 944-955.

74 R. Wieczorek, L. Haskamp and J. J. Dannenberg, J. Phys. Chem. A, 2004, 198, 6713-6723.

75 J. M. Berg, J. L. Tymoczko and L. Stryer, Biochemistry, W.H. Freeman, Basingstoke, 7th edn, 2012. 